Aim of the study: Thyroid carcinoma is the most common malignancy of endocrine organs. The prognosis varies. Factors such as age, sex, size of the tumor, stage of disease, presence of extrathyroidal spread, and completeness of resection have been found to significantly influence prognosis. We aimed to evaluate clinical features of our patients with thyroid carcinoma, prospectively. Material and methods: We evaluated total 178 patients treated between 2010 and 2011 at the Department of Endocrinology, İzmir Atatürk Training Hospital, retrospectively. Data on patients, tumors, and therapeutic approaches were collected. All results are shown as mean \pm standard deviation (SD). $P$ values were based on two-sided tests with a cutoff for statistical significance of 0.05 and $95 \%$ confidence interval. Results: There were no differences between female and male patients according to histopathological subtypes, demographic data and prognostic findings of thyroid cancer. The assessment of tumor size and other prognostic factors revealed that there was a correlation between tumor size and capsular and/or vascular invasion. In the postoperative evaluation we detected a correlation between metastases and vascular invasion and/or capsular invasion but there was no significant relation between focus (solitary/multifocal) and metastases.

Conclusion: There was no significant difference in terms of gender and age ( $<45$ years of age and $\geq 45$ years of age) among the patient groups (low risk/intermediate risk/high risk). By multiple regression analysis among metastasis and prognostic factors it was observed that vascular invasion and thyroglobulin levels affect development of metastases.

Key words: differentiated thyroid carcinoma, papillary thyroid carcinoma, thyroglobulin, TNM, tumor, node, metastases.

\section{Clinical experience of thyroid carcinoma: a study of 178 cases}

\author{
Harman Ece ${ }^{1}$, Cetinkaya Dölek Devrim ${ }^{1}$, Tutuncuoglu A. Pelin ${ }^{1}$ \\ Gorgel Ahmet ${ }^{1}$, Niflıoglu M. Ozgur ${ }^{2}$, Harman Mustafa ${ }^{3}$, Bahceci Mithat ${ }^{1}$
}

\author{
${ }^{1}$ Department of Endocrinology and Metabolism Disease, Izmir Ataturk Training \\ and Research Hospital, Izmir, Turkey \\ 2Department of Internal Medicine, Izmir Ataturk Training and Research Hospital, \\ Izmir, Turkey \\ ${ }^{3}$ Department of Radiology, Ege University Medical Faculty, Izmir, Turkey
}

\section{Introduction}

Although thyroid cancer is a rare disease, its incidence increases with age and use of diagnostic methods such as ultrasound guided fine needle aspiration biopsy. In countries with sufficient iodine supply or only moderate iodine deficiency, papillary thyroid cancer is the dominant histopathological form and accounts for more than $70 \%$. Differentiated thyroid cancers are two to four times as common in females as in males; however, the female preponderance decreases in prepubertal and postmenopausal ages, which suggests that sex hormones might play some role in the pathogenesis.

Prognosis of thyroid cancer is good in general and patients with differentiated thyroid cancer have 10-year cancer-specific mortality rates of less than $10 \%$ [1]. On the other hand, prognostic factors may affect survival of patients. The most important prognostic factors in well-differentiated thyroid carcinoma are age (older than 45 years) and gender (male patients). The recurrence rates are reported in the vicinity of $8 \%$ to $23 \%$ [2, 3]. The AMES (age, distant metastasis, tumor extent, and size), AGES (age, tumor size, histological grade, tumor extent, distant metastasis) and MACIS (distant metastasis, age, completeness of primary tumor resection, local invasion, and tumor size) prognostic systems for well-differentiated thyroid carcinoma are well known [4].

Histopathological variants of papillary thyroid cancer may also affect the prognosis of the disease. In fact, tall cell variant of papillary thyroid cancer is a more aggressive variant than classical papillary thyroid cancer and has a poor prognosis. The diffuse sclerosing variant of papillary thyroid cancer predominantly observed in young patients is a rare aggressive tumor that requires intensive treatment [5].

The approach to risk assessment of thyroid cancer is very important. American Thyroid Association (ATA) guidelines are used for evaluation of these cases [6]. Three risk groups (low, intermediate and high risk) have been reported according to ATA guidelines.

In this study we aimed to investigate clinical characteristics and prognostic factors of all thyroid cancer cases, prospectively.

\section{Material and methods}

\section{Patients}

This study was carried out in İmir Atatürk Training Hospital, Department of Endocrinology. All patients with thyroid cancer $(n=178)$ between 2010 and 2011 were enrolled in the study prospectively and respectively.

\section{Methods}

All data about risk status such as age, sex, tumor data (pathological diagnosis, size, multifocal/solitary, stage, extrathyroidal spread), metastasis 
(locoregional, distant), mode of detection (clinical, imaging, thyroglobulin estimation), and treatment modalities (surgery, iodine 131 therapy) were recorded. Treatment success was determined on the basis of undetectable thyroglobulin estimation under TSH elevation (after withdrawal of thyroid hormone over the course of 4-6 weeks) and/or normalization of imaging modalities including thyroid ultrasonography (USG), thyroid scintigraphy, thorax computed tomography (CT), whole body scintigraphy, and abdominal USG.

\section{Diagnosis of thyroid cancer}

Ultrasound-guided fine needle aspiration biopsy was performed in all 178 patients. 112 patients were diagnosed with FNAB; and the remaining 66 patients were diagnosed postoperatively, although they were benign $(n=13)$, suspicious cytology $(n=51)$ and non-diagnostic cytology $(n=2)$ in FNAB.

\section{Biochemical analysis}

Biochemical parameters (TSH, FT3 and FT4 hormones) were evaluated with chemiluminescence analyzers by Advia Centaur XR Hormone Analyzer. Calcitonin and thyroglobulin levels were detected with chemiluminescence analyzers by Immulate 2000 Hormone Analyzer.

\section{Statistical analysis}

All results are shown as mean \pm standard deviation (SD). $P$ values were based on two-sided tests with a cutoff for statistical significance of 0.05 and 95\% confidence interval. The Kolmogorov-Smirnov test, $\chi^{2}$ test, Fisher's exact test, MannWhitney $U$ test, independent sample t test and analysis of covariance test (ANOVA) were used to evaluate values. All statistical analyses were performed with MedCalc Statistical Software Version 10.1.6.0 (Licensed to MedCalc Turkey 020931118117) and SPSS (Statistical Package for Social Sciences) version 15.0.

\section{Results}

\section{Demographic data and prognostic findings} of the patients

Our patients had a clear female preponderance $(85.4 \%$ vs. $14.6 \%, p=0.001)$. Mean age of all patients was $48.5 \pm 24.0$ years, and $48.2 \pm 25.4$ years in female and $50.1 \pm 12.4$ in male patients, respectively. Mean age of female and male patients was similar. In terms of poor prognostic parameters such as focus, capsular invasion, metastases and tumor size, there were no significant differences between male and female patients. Of 178 patients, 85 patients had macrocarcinoma (> $10 \mathrm{~mm}$ ) and 93 patients had microcarcinoma (<10 mm). We detected that 18 patients had distant metastases (hepatic metastasis in 1 patient, pulmonary metastases in 16 patients, bone metastasis in 1 patient), 30 patients had vascular invasion, and 63 patients had capsular invasion. All data of demographic and prognostic findings are shown in Table 1.

In our study, there were no differences between female and male patients in terms of histopathological subtypes of thyroid cancer (Table 2). Two of the 16 patients with thyroid medullary cancer had metastases and significantly high serum calcitonin levels were detected $(p<0.05)$. There were normal serum calcitonin levels in the patients who had thyroid medullary carcinoma without metastases. Similarly, there were normal serum calcitonin levels in the patients with papillary cancer + medullary cancer (mix). We detected pheochromocytoma and primary hyperparathyroidism in 2 female patients with medullary cancer. They were diagnosed as MEN II A syndrome.

\section{Assessment of relationship between tumor size and the other prognostic findings}

We evaluated the relationship between tumor size and poor prognostic factors and as a result we found that there was a positive correlation between tumor size and capsular and/or vascular invasion (capsular invasion, $r=0.54$, $p<0.05$; vascular invasion, $r=0.44, p<0.05$ ). In addition, we detected a statistically significant relation between focality (solitary/multifocal) and capsular invasion, similarly between vascular invasion and capsular invasion (Table 3). In evaluation of patients with metastases, there was no significant correlation between metastases and focus (solitary/multifocal) but there was a statistically significant relation between metastases and tumor size, capsular invasion, and vascular invasion $(p<0.05)$.

Table 1. Demographic data and prognostic findings of the patients

\begin{tabular}{|c|c|c|c|c|c|}
\hline & & Male $(n=26)$ & Female $(n=152)$ & Total $(n=178)$ & $p$ value \\
\hline \multirow[t]{2}{*}{ Focus } & solitary & 19 (73.1\%) & 119 (78.3\%) & 138 (77.5\%) & \multirow{2}{*}{ NS } \\
\hline & multifocal & 7 (26.9\%) & $33(21.7 \%)$ & 40 (22.5\%) & \\
\hline \multirow[t]{2}{*}{ Capsular invasion } & + & $9(34.6 \%)$ & $54(35.5 \%)$ & $63(35.4 \%)$ & \multirow{2}{*}{ NS } \\
\hline & - & $1765.4 \%)$ & 98 (64.5\%) & 115 (64.6\%) & \\
\hline \multirow[t]{2}{*}{ Vascular invasion } & + & $6(23.1 \%)$ & $24(15.8 \%)$ & 30 (16.9\%) & \multirow{2}{*}{ NS } \\
\hline & - & 20 (76.9\%) & $128(84.2 \%)$ & $148(83.1 \%)$ & \\
\hline \multirow[t]{2}{*}{ Metastases } & + & $4(15.4 \%)$ & 14 (9.2\%) & $18(10.1 \%)$ & \multirow{2}{*}{ NS } \\
\hline & - & $22(84.6 \%)$ & $138(90.8 \%)$ & 160 (89.9\%) & \\
\hline \multirow[t]{2}{*}{ Tumor size } & $\leq 10.0 \mathrm{~mm}$ & 12 (46.2\%) & 81 (53.3\%) & $93(52.2 \%)$ & \multirow{2}{*}{ NS } \\
\hline & $>10.0 \mathrm{~mm}$ & $14(53.8 \%)$ & 71 (46.7\%) & 85 (47.8\%) & \\
\hline
\end{tabular}


Table 2. Histopathological analysis of female and male patients

\begin{tabular}{|c|c|c|c|c|}
\hline $\begin{array}{l}\text { Histopathological } \\
\text { diagnosis }\end{array}$ & Female $(n=152)$ & Male $(n=26)$ & Total $(n=178)$ & $P$-value* \\
\hline papillary microcancer & $66(43.4 \%)$ & $11(42.3 \%)$ & 77 (43.3\%) & NS \\
\hline papillary cancer follicular variant & $16(10.5 \%)$ & $2(7.7 \%)$ & $18(10.1 \%)$ & NS \\
\hline papillary cancer oncocytic variant & $1(0.7 \%)$ & $0(0.0 \%)$ & $1(0.6 \%)$ & NS \\
\hline papillary cancer classical variant & $38(25.0 \%)$ & $6(23.1 \%)$ & $44(24.7 \%)$ & NS \\
\hline minimally invasive follicular carcinoma & $5(3.3 \%)$ & $0(0.0 \%)$ & $5(2.8 \%)$ & NS \\
\hline follicular carcinoma & $4(2.6 \%)$ & $2(7.7 \%)$ & $6(3.3 \%)$ & NS \\
\hline thyroid medullary cancer & $13(8.6 \%)$ & $3(11.5 \%)$ & $16(9.0 \%)$ & NS \\
\hline hurthle cell carcinoma & $1(0.7 \%)$ & $0(0.0 \%)$ & $1(0.6 \%)$ & NS \\
\hline follicular adenoma & $1(0.7 \%)$ & $0(0.0 \%)$ & $1(0.6 \%)$ & NS \\
\hline papillary cancer + thyroid medullary cancer (mix) & $2(1.3 \%)$ & $0(0.0 \%)$ & $2(1.1 \%)$ & NS \\
\hline follicular cancer oncocytic variant & $2(1.3 \%)$ & $1(3.8 \%)$ & $3(1.7 \%)$ & NS \\
\hline papillary cancer + follicular cancer (mix) & $1(0.7 \%)$ & $1(3.8 \%)$ & $2(1.2 \%)$ & NS \\
\hline undifferentiated thyroid cancer & $2(1.3 \%)$ & $0(0.0 \%)$ & $2(1.1 \%)$ & NS \\
\hline
\end{tabular}

${ }^{*} N S$ - non-significant

Table 3. Relationship between tumor size and the other prognostic findings

\begin{tabular}{|c|c|c|c|c|c|}
\hline & \multicolumn{2}{|c|}{ Focus } & \multirow{2}{*}{$\begin{array}{l}\text { Capsular } \\
\text { invasion }\end{array}$} & \multirow{2}{*}{$\begin{array}{l}\text { Vascular } \\
\text { invasion }\end{array}$} & \multirow[t]{2}{*}{ Total } \\
\hline & Solitary & Multifocal & & & \\
\hline < 10 mm tumor size & $75(80.6 \%)$ & $18(19.4 \%)$ & $10(10.8 \%)$ & $1(1.1 \%)$ & $93(52.2 \%)$ \\
\hline$>10$ mm tumor size & $63(74.1 \%)$ & $22(25.9 \%)$ & $53(62.4 \%)$ & $29(34.1 \%)$ & $85(47.8 \%)$ \\
\hline total & $138(77.5 \%)$ & $40(22.5 \%)$ & $63(35.4 \%)$ & $30(16.9 \%)$ & $178(100 \%)$ \\
\hline$p$-value & $p>0.05$ & $p>0.05$ & $P<0.05^{*}$ & $P<0.05^{*}$ & \\
\hline
\end{tabular}

\section{Distribution of patients according to risk group}

All patients were divided into 3 groups according to their risk level based on ATA guidelines as low risk (group I), intermediate risk (group II) and high risk (group III) [6]. There were 161 patients in group I [138 female (85.7\%), 23 male (14.3\%)], 15 patients in group II [12 female (80\%), 3 male (20\%)] and only 2 patients in group III (1 female, 1 male). Gender distribution in all risk groups was similar to the whole patient population. Significant differences could not be detected in terms of gender and age ( $<45$ years of age and $\geq 45$ years of age) among groups I, II and III.

\section{Postoperative screening and evaluation}

We detected residual thyroid tissue in 55 patients, residual thyroid tissue with recurring thyroid nodule in 4 patients, and residual tissue with lymphadenopathy in 19 patients by ultrasonographic examination. Tg levels between the patients with and without residual thyroid tissue were significantly different (respectively, $n=87$, $\operatorname{tg}=3.74 \pm 5.89$; $n=91, \operatorname{tg}=1.47 \pm 2.62 ; p=0.01$ ). In postoperative scanning (ultrasonography, thyroid scintigraphy, computed tomography, whole body scintigraphy), we detected 18 patients with metastases (hepatic metastasis in 1 patient, pulmonary metastases in 16 patients, bone metastases in 1 patient). In terms of thyroglobulin levels there was a significant difference between the patients with and without metastases (respectively, $n=18, \operatorname{tg}=2.51 \pm 3.54 ; n=160, \operatorname{tg}=2.58 \pm 4.75 ; p>0.05)$.

\section{Discussion}

Thyroid cancer is one of the fastest growing cancer diagnoses worldwide. It is 2.9 times more common in women than men (female : male ratio 16.3 : 5.7) [7]. In our study, we observed that our patients had a clear female preponderance too. The cause of this gender discrepancy is not clear yet. Many factors have been evaluated in terms of dominance by gender in thyroid cancer. The fluctuation of sex hormones during women's menstrual cycle and pregnancy has been hypothesized as the reason for gender disparity in papillary thyroid cancer. In particular, the peak incidence of papillary thyroid cancer has been observed in women aged $40-49$ years, this being the age group at which most women approach or enter menopause [7-9]. There were also several studies looking at the association of papillary thyroid cancer with reproductive factors such as age of menarche, menopause, number of pregnancies and other parameters [9-14]. Effects of sex hormone are mediated by hormone-specific nuclear receptors and the 
effects of estrogen on thyroid cancer cell lines are dependent on the type of thyroid cancer, and estrogen dramatically increases estrogen receptor $\alpha$ levels in papillary thyroid cancer, whereas in anaplastic thyroid cancer and follicular thyroid cancer the receptor levels are not significantly altered [15-17]. In this study, mean age of female and male patients was similar. We can claim that there is no difference between male and female patients in terms of age. In females, the agespecific incidence rate rises sharply at the beginning of the reproductive years, with increasing age peaking at 40-49 years, while in the men the peak is at 60-69 years. It was shown that the incidence rates equalize by 85 years of age $[7,8]$.

It was reported that less aggressive histological subtypes of thyroid cancer were more common in women, whereas the more aggressive types of thyroid cancer, anaplastic thyroid cancer and medullary thyroid cancer, have similar rates of incidence in men and women. In contrast we could not determine any significant difference between female and male patients in terms of histopathological subtypes of thyroid cancer. We served as a reference hospital and our patients were selected and referred patients. This situation may be responsible for this result.

It was detected that male sex, advanced initial stage, and presence of extrathyroidal spread within the primary tumor are the most significant independent predictors of developing multiple recurrences in patients with well-differentiated thyroid cancer [18]. Factors such as age, sex, size of the tumor, stage of disease, presence of extrathyroidal spread, and completeness of resection have also been found to significantly influence prognosis [19]. However, in our study we could not find any significant difference in terms of gender and age ( $<45$ years of age and $\geq 45$ years of age) in the patients with poor prognostic factors.

We detected 93 patients with microcancer $(<10 \mathrm{~mm})$ There were solitary cancer $(n=75)$ and multifocal cancer $(n=18)$. In these patients with thyroid microcarcinoma, 10 patients had capsular invasion, 1 patient had vascular invasion, and 2 patients had metastasis. Among the patients with thyroid micropapillary cancer $(n=83)$ we detected capsular invasion $(n=5)$, vascular invasion $(n=1)$, solitary cancer $(n=67)$, multifocal cancer $(n=16)$, and metastases $(n=2)$. In accordance with these results we can claim that thyroid microcarcinomas may not be as innocent as they say. An increasing proportion of newly diagnosed thyroid carcinomas have a small size: when no larger than $1.0 \mathrm{~cm}$ in diameter, they are classified as microcarcinomas. Many microcarcinomas may remain occult and become diagnosed as an incidental finding during surgery for goiter or other benign thyroid disorders. Microcarcinomas in multicentric papillary thyroid cancer should be treated as high-risk tumors [20]. In autopsy series the rate of papillary microcancer (PMC) varies from $1 \%$ to $35 \%$ [21]. In some studies, it was reported that $20 \%$ of patients had PMC [21]. Patients with PMC $(n=203)$ were investigated retrospectively and it was found that the disease-related mortality rate was $1.0 \%$, lymph node recurrence was 5.0\%, and the distant metastases rate was 5.0\% [22]. In our study, there were 85 patients with thyroid macrocancer (> $10 \mathrm{~mm}$ ). Among these patients we detected multifocal cancer $(n=22)$, solitary cancer $(n=63)$, capsular invasion $(n=53)$, vascular invasion $(n=29)$, and metastases $(n=15)$. In the patients with papillary macrocancer $(n=56)$ we found solitary cancer $(n=40)$, multifocal cancer $(n=16)$, capsular invasion $(n=31)$, vascular invasion $(n=17)$, and metastases $(n=11)$.

We found that there was a positive correlation between tumor size and capsular and/or vascular invasion. In addition, we detected a statistically significant relation between focality and capsular invasion. In evaluation of patients with metastases, there was no significant correlation between metastases and focus (solitary/multifocal) but there was a statistically significant relation between metastases and tumor size, capsule invasion, and vascular invasion.

Generally, it was reported that there were distant metastases for patients with tumors greater than $1.5 \mathrm{~cm}$ [2]. In one study, there was no relation between tumor size and distant metastases [23]. Some authors emphasized that bilateral foci were associated with tumor size larger than $1.0 \mathrm{~cm}$. In the same study, extrathyroidal invasion was found to be significantly associated with male sex, vascular invasion, lymph node metastases, and tumor size larger than $1.0 \mathrm{~cm}$. Moreover, there were significant relations between lymph node metastases and other prognostic factors (patient age above 45 years, extrathyroidal invasion) [24]. A clear association was identified between extrathyroidal spread and the development of both locoregional and distant recurrence $[25,26]$.

In this study, there were no differences between female and male patients in terms of prognostic factors. A significant difference was not detected according to age ( $<45$ years of age and $\geq 45$ years of age) among risk groups. There was a significant relation between extrathyroidal spread and distant metastases. The tumor size were found to be significantly large in the patients with distant metastases and lymph node metastases. Furthermore, in terms of histopathological assessment, there was no significant statistical difference between the ones with or without metastases. The most commonly used risk stratification systems, such as MACIS (metastases, age, completeness of resection, invasion, and size) and the TNM system of the American Joint Committee on Cancer (AJCC), were designed to predict disease-specific mortality rather than risk of recurrence $[27,28]$. This has resulted in the recognition of low- and high-risk patient categories and allowed meaningful comparison of a variety of treatment approaches. Tuttle et al. stratified risk of death into four categories: very low risk, low risk, intermediate risk, and high risk. High-risk features are age $>45$ years, larger tumors (> $4 \mathrm{~cm}$ ) or worrisome histology, incomplete resection, distant metastases, and cervical lymph node metastases. Low-risk features are young age, classical histology, smaller tumors, complete resection, no distant metastases, and no cervical lymph node involvement (in patients $<45$ years of age, lymph node involvement did not correlate with increased risk of death). Intermediate-risk disease includes young patients with classic papillary tumors $>4 \mathrm{~cm}$, microscopic extrathyroidal disease, more aggressive histology or vascular invasion, or older patients with classic papillary histology with a size $<4 \mathrm{~cm}$, or extrathyroidal extension, aggressive histology $<1 \mathrm{~cm}$ to $2 \mathrm{~cm}$ but with complete resection and without distant metastases [29]. Thus, the treatment plan must be created after the risk assessment is done. For each individual patient, the physician must rely on clinical judg- 
ment and consider the disease and the patient's wishes before deciding on the management strategy.

\section{Acknowledgments}

We have no conflict of interest in this study. Written informed consent was taken from all the subjects before participating.

I am indebted to many of my colleagues who supported me in this study.

\section{References}

1. HundahI SA, Fleming ID, Fremgen AM, Menck HR. A National Cancer Data Base report on 53,856 cases of thyroid carcinoma treated in the U.S., 1985-1995. [see commetns]. Cancer 1998; 83: 2638 48..

2. Mazzaferri EL, Jhiang SM. Long-term impact of initial surgical and medical therapy on papillary and follicular thyroid cancer. Am J Med 1994; 97: 418-28.

3. Samaan NA, Schultz PN, Hickey RC, Goepfert H, Haynie TP, Johnston DA Ordonez NG. The results of various modalities of treatment of well differentiated thyroid carcinomas: a retrospective review of 1599 patients. J Clin Endocrinol Metab 1992; 75: 714-20.

4. Mazzaferri EL. An overview of the management of papillary and fol licular thyroid carcinoma. Thyroid 1999; 9: 421-7.

5. Lin JD, Hsueh C, Huang BY. Papillary thyroid carcinoma with different histological patterns. Chang Gung Med J 2011; 34: 23-34.

6. American Thyroid Association (ATA) Guidelines Taskforce on Thyroid Nodules and Differentiated Thyroid Cancer; Cooper DS, Doher ty GM, Haugen BR, et al. Revised American Thyroid Association management guidelines for patients with thyroid nodules and differentiated thyroid cancer. Thyroid 2009; 19: 1167-214.

7. Ortega J, Sala C, Flor B, Lledo S.Efficacy and cost-effectiveness of the UltraCision harmonic scalpel in thyroid surgery: an analysis of 200 cases in a randomized trial. J Laparoendosc Adv Surg Tech A 2004; 14: 9-12.

8. Kilfoy BA, Devesa SS, Ward MH, Zhang Y, Rosenberg PS, Holford TR, Anderson WF. Gender is an age-specific effect modifier for papillary cancers of the thyroid gland. Cancer Epidemiol Biomarkers Prev 2009; 18: 1092-100.

9. Negri E, Dal Maso L, Ron E, et al. A pooled analysis of case-control studies of thyroid cancer. II. Menstrual and reproductive factors. Cancer Causes Control 1999; 10: 143-55.

10. Brindel P, Doyon F, Rachédi F, Boissin JL, Sebbag J, Shan L, et al. Menstrual and reproductive factors in the risk of differentiated thyroid carcinoma in native women in French Polynesia: a population-based case-control study. Am J Epidemiol 2008; 167: 219-29

11. Mack WJ, Preston-Martin S, Bernstein L, Qian D, Xiang M. Reproductive and hormonal risk factors for thyroid cancer in Los Angeles County females. Cancer Epidemiol Biomarkers Prev 1999; 8: 991-7.

12. Pham TM, Fujino Y, Mikami H, Okamoto N, Hoshiyama Y, Tamakoshi A, Matsuda S,Yoshimura T; Japan Collaborative Cohort Study Group. Reproductive and menstrual factors and thyroid cancer among Japanese women: the Japan Collaborative Cohort Study. J Womens Health (Larchmt) 2009; 18: 331-5.

13. Sakoda LC, Horn-Ross PL. Reproductive and menstrual history and papillary thyroid cancer risk: the San Francisco Bay Area thyroid cancer study. Cancer Epidemiol Biomarkers Prev 2002; 11: 51-7.

14. Truong T, Orsi L, Dubourdieu D, Rougier Y, Hémon D, Guénel P. Role of goiter and of menstrual and reproductive factors in thyroid cancer: a population-based case-control study in New Caledonia (South Pacific), a very high incidence area. Am J Epidemiol 2005; 161 1056-65.

15. Yager JD, Liehr JG. Molecular mechanisms of estrogen carcinogenesis. Annu Rev Pharmacol Toxicol 1996; 36: 203-32.

16. Zeng Q, Chen GG, Vlantis AC, van Hasselt CA. Oestrogen mediates the growth of human thyroid carcinoma cells via an oestrogen receptor-ERK pathway. Cell Prolif 2007; 40: 921-35.
17. Zeng $\mathrm{O}$, Chen $\mathrm{G}$, Vlantis A, Tse $\mathrm{G}$, van Hasselt C. The contributions of oestrogen receptor isoforms to the development of papillary and anaplastic thyroid carcinomas. J Pathol 2008; 214: 425-33.

18. Palme CE, Waseem Z, Raza SN, Eski S, Walfish P, Freeman JL. Management and outcome of recurrent well-differentiated thyroid carcinoma. Arch Otolaryngol Head Neck Surg 2004; 130: 819-24.

19. Mazzaferri EL. Papillary thyroid carcinoma: factors influencing prognosis and current therapy. Semin Oncol 1987; 14: 315-32.

20. Lin JD, Chao TC, Hsueh C, Kuo SF. High recurrent rate of multicentric papillary thyroid carcinoma. Ann Surg Oncol 2009; 16: 2609-16.

21. Yamamoto Y, Maeda T, Izumi K, Otsuka H. Occult papillary carcinoma of the thyroid. A study of 408 autopsy cases. Cancer 1990; 65: $1173-9$.

22. Chow SM, Law SC, Chan JK, Au SK, Yau S, Lau WH. Papillary microcarcinoma of the thyroid-Prognostic significance of lymph node metastasis and multifocality. Cancer 2003; 98: 31-40.

23. Brierley J, Tsang R, Panzarella T, Bana N. Prognostic factors and the effect of treatment with radioactive iodine and external beam radiation on patients with differentiated thyroid cancer seen at a single institution over 40 years. Clin Endocrinol (Oxf) 2005; 63: 418-27.

24. Pellegriti G, Scollo C, Lumera G, Regalbuto C, Vigneri R, Belfiore A. Clinical behavior and outcome of papillary thyroid cancers smaller than $1.5 \mathrm{~cm}$ in diameter: study of 299 cases. J Clin Endocrinol Metab 2004; 89: 3713-20.

25. McConahey WM, Hay ID, Woolner LB, van Heerden JA, Taylor WF. Papillary thyroid cancer treated at the Mayo Clinic, 1946 through 1970: initial manifestations, pathologic findings, therapy, and outcome. Mayo Clin Proc 1986; 61: 978-96.

26. Andersen PE, Kinsella J, Loree TR, Shaha AR, Shah JP. Differentiated carcinoma of the thyroid with extrathyroidal extension. Am J Surg 1995; 170: 467-70.

27. Singletary SE, Allred C, Ashley P, et al. Staging system for breast cancer: revisions for the 6th edition of the AJCC Cancer Staging Manual. Surg Clin North Am 2003; 83: 803-19.

28. Hay ID, Bergstralh EJ, Goellner JR, Ebersold JR, Grant CS. Predicting outcome in papillary thyroid carcinoma: development of a reliable prognostic scoring system in a cohort of 1779 patients surgically treated at one institution during 1940 through 1989. Surgery 1993; 114: 1050-7.

29. Carter WB, Tourtelot JB, Savell JG, Lilienfeld H. New treatments and shifting paradigms in differentiated thyroid cancer management. Cancer Control 2011; 18: 96-103.

\section{Address for correspondence}

\section{Ece Harman MD}

Department of Endocrinology and Metabolism Disease Izmir Ataturk Training and Research Hospital

35290 Izmir, Turkey

tel. +90 5372545328

$\mathrm{fax}+902322431530$

e-mail: ecarmu@gmail.com

Submitted: $\quad 6.02 .2012$

\section{Accepted: $\quad 19.032012$}

